\title{
Poules et poulets entre métaphores et realia dans la prédication et les exempla du Moyen Âge
}

Hens and chickens between metaphors and realia in Medieval predication and exempla

Marie Anne Polo de Beaulieu

\section{OpenEdition}

\section{Journals}

Édition électronique

URL : http://journals.openedition.org/ethnoecologie/3335

DOI : 10.4000/ethnoecologie.3335

ISSN : 2267-2419

Éditeur

Laboratoire Eco-anthropologie et Ethnobiologie

Référence électronique

Marie Anne Polo de Beaulieu, « Poules et poulets entre métaphores et realia dans la prédication et les exempla du Moyen Âge », Revue d'ethnoécologie [En ligne], 12 | 2017, mis en ligne le 18 décembre 2017, consulté le 19 avril 2019. URL : http://journals.openedition.org/ethnoecologie/3335 ; DOI : 10.4000/ ethnoecologie.3335

Ce document a été généré automatiquement le 19 avril 2019

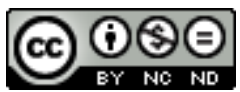

Revue d'ethnoécologie est mis à disposition selon les termes de la licence Creative Commons Attribution - Pas d'Utilisation Commerciale - Pas de Modification 4.0 International. 


\title{
Poules et poulets entre métaphores et realia dans la prédication et les exempla du Moyen Âge
}

Hens and chickens between metaphors and realia in Medieval predication and exempla

\author{
Marie Anne Polo de Beaulieu
}

1 Les exempla, ces anecdotes exemplaires qui fleurissent dans la prédication et la littérature morale au tournant du XIII ${ }^{\mathrm{e}}$ siècle (Welter 1927, Le Goff et al. 1982-1996, Polo de Beaulieu \& al. 2010), sont-ils la bible de la vie quotidienne ? Oui, car, tout comme la bible, les exempla, instruments de travail du prédicateur en amont de la prédication, représentent un réservoir de récits et de connaissances tout autant que de croyances. Les exempla vulgarisent ce savoir biblique, mais il n'est jamais nu, toujours enveloppé de commentaires et réécritures, voire de traductions en langue vernaculaire, et enjolivé d'apocryphes, sans cesse renouvelés. On peut considérer, à l'inverse, que les exempla ne sont pas la bible de la vie quotidienne dans la mesure où ils offrent des apparences de realia mais toujours soumises à une moralisation qui peut distordre la représentation des réalités sociales, comme l'ont montré le volume dédié à L'animal exemplaire (Berlioz \& Polo de Beaulieu 1999) et l'étude particulière consacrée aux oiseaux dans les exempla monastiques (Berlioz \& Cordonnier 2004) et à la cigogne adultère (Berlioz 2002).

2 L'entrée en scène des animaux dans la prédication est à mettre en relation avec leur importance dans l'hagiographie et la scolastique à la fin du XIII ${ }^{\mathrm{e}}$ siècle. Pierre Boglioni (Boglioni 1999:51-80, spéc. 63) a montré la proximité des saintes de cette période avec les animaux, notamment la bienheureuse Ide de Louvain (v. 1212-1262), dont la Vita est parsemée de scènes joyeuses avec les animaux : des poissons (I, 29), des coqs et des poules (I, 30), dans une tonalité franciscaine marquée (BHL, 4145 ; AASS, apr. II, 158-189). Cette même influence franciscaine - plus précisément du courant de pensée autour de Matthieu d'Aquasparta, de John Pekham et de Roger Marston - explique, selon Alain Boureau, le retour éphémère des animaux dans la scolastique des années 1270-1280 (Boureau 1999: 
107-108), grâce à une conjonction avec le savoir naturaliste, notamment dans le corpus d'Avicenne. Pour montrer le pouvoir propre de la volonté et de l'imagination sur le monde réel, il est fait précisément recours à la poule d'Avicenne (De animalibus : VIII, 7) :

«La poule, quand elle l'emporte sur le coq dans un combat, se comportera comme un coq et parfois lui pousse un ergot sur la patte comme il en existe chez le coq; on peut alors saisir l'obéissance de la matière naturelle aux pensées de l'âme ».

Ce fait prouve, selon les scolastiques, que les animaux partagent avec les hommes le pouvoir de l'énergie imaginante.

4 Cependant, cette poule d'Avicenne n'est pas reprise dans les sermons et les exempla, qui se placent dans une perspective différente. Dans cette production pastorale aussi abondante qu'ambiguë, quelles images de la poule (gallina) et du poulet (pullus) l'historien peut-il trouver?

5 Pour répondre à ce questionnement, nous examinerons des recueils d'exempla mais également les exempla inclus dans les sermons diffusés par toutes sortes de prédicateurs : clercs séculiers, moines cisterciens, frères dominicains et franciscains. En général conservés en latin, ces exempla avaient vocation à être insérés dans des sermons prononcés en langue vulgaire dans la prédication au peuple (ad populum), mais en latin pour les clercs (ad cleros), notamment ceux de l'université.

\section{Présentation des sources}

Nos sources peuvent s'inspirer du Physiologus, encyclopédie composée au II ${ }^{\mathrm{e}}$ siècle apr. J.C. par un auteur anonyme à Alexandrie. L'ouvrage mêle des citations bibliques, des légendes au savoir naturaliste antique. Les caractéristiques des animaux y sont moralisées. L'immense succès de cette encyclopédie explique ses emprunts dans les bestiaires qui lui adjoignent des extraits de l'Hexaméron d'Ambroise de Milan et des Étymologies d'Isidore de Séville. Les bestiaires moralisés ajoutent parfois une nouvelle lecture allégorique moralisante. Ils s'inscrivent dans la droite ligne du néoplatonisme augustinien, pour lequel toute créature reflète le créateur. La création est conçue comme un univers de signes à déchiffrer. Les plantes, les minéraux et les animaux participent donc de cette sémiologie chrétienne. Le monde de la fable n'est pas inconnu non plus des prédicateurs, notamment mendiants (Boivin et al. 2011).

7 Nous examinerons des exempla issus de sermons ou de recueils composés aux XIII ${ }^{\mathrm{e}}$ et XIV siècles par des clercs séculiers comme Jacques de Vitry (Sermones Vulgares vel ad status, Crane 1890, Longère 2013) ${ }^{1}$ ou Eudes de Cherinton (Longère 1982) ${ }^{2}$, mais également par un moine cistercien, Césaire de Heisterbach (McGuire 2004, Smirnova et al. 2015) ${ }^{3}$. La part belle sera faite aux ordres mendiants, notamment les dominicains, les plus prolifiques dans la rhétorique exemplaire: Jacques de Voragine, archevêque de Gênes (Bertini Guidetti 1998) ${ }^{4}$, Étienne de Bourbon, inquisiteur (Berlioz éd. 2002, 2006, 2015) ${ }^{5}$, Thomas de Cantimpré, proche des Béguines du Brabant (Platelle 1997) ${ }^{6}$, Jean Gobi le Jeune, lecteur du couvent royal de Saint-Maximin en Provence (Polo de Beaulieu 1999) ${ }^{7}$ et Filippe de Ferrare (Creytens 1946, Amadori 1996, Vecchio 1998) ${ }^{8}$, sans oublier une compilation anonyme d'origine franciscaine : la Tabula exemplorum (Welter (Ed.) 1927) ${ }^{9}$ et les sermons du célèbre saint Bernardin de Sienne (Delcorno 1989) dont les sermons mobilisaient d'immenses foules en Italie ${ }^{10}$. Enfin, les exempla en ancien français seront également pris en considération, grâce au riche mais anonyme recueil intitulé le $\mathrm{Ci}$ nous dit (Blangez 
1979-1986) dont la diffusion aristocratique par la lecture individuelle ou collective correspond plutôt à ce que Michel Zink appelait la « prédication dans un fauteuil » (Zinc $1976: 478)^{11}$.

8 Tous ces textes vont nous permettre de saisir l'imaginaire attaché à la poule, comparée aux autres oiseaux, d'observer sa mise en scène dans un contexte social proche des realia, puis la place de ce volatile dans le savoir naturaliste moralisé inspiré des bestiaires et enfin l'allégorisation extrême de la poule et du poulet qui peuvent représenter la Vierge et le Christ dans quelques sermons et exempla.

\section{Imaginaire de la poule et classement hiérarchique des oiseaux}

9 Pour introduire la leçon selon laquelle si les riches profitent de tous les bienfaits dans la vie présente, ils ne sont que de vils cadavres après leur mort, leçon illustrée par le récit devenu célèbre sous le titre du Marchand de Venise (Shakespeare), Jacques de Vitry compare la vie et la mort de la poule et du faucon: «Le faucon choyé de son vivant est jeté au fumier une fois mort. La poule, vivante, gratte le fumier; morte, elle est sur la table des rois. » (Crane $1890:$ n. 47). Cet exemplum illustre un sermon destiné aux moines noirs, c'est-à-dire les Bénédictins (Longère 2013 : 415).

10 L'assimilation entre les riches et le faucon, et les pauvres et la poule instille un catéchisme social très présent par la suite dans les sermons des frères mendiants. C'est ainsi que l'on retrouve la même comparaison, légèrement différente, sous la plume du dominicain Filippe de Ferrare, dans son Manuel de conversation (Vecchio $1998: \mathrm{n}$. 78) : « La poule maltraitée pendant sa vie est appréciée après la mort alors que le faucon apprécié durant sa vie est maltraité après la mort. La poule pendant toute sa vie est maltraitée : elle n'a pas le droit d'entrer dans la maison et dans le cas contraire, elle en est chassée violemment. Au contraire, le faucon est gardé dans la maison avec tous les honneurs : on le place sur une barre et on lui donne à manger des bons morceaux de viande. Une fois morte, la poule est portée devant le chef de la maison avec tous les honneurs ; à l'inverse, une fois mort, le faucon est jeté avec les ordures. »

11 Cette reformulation connut un grand succès attesté par de nombreuses copies ou emprunts (TheMA). Ces deux exemples donnent à voir des realia: la poule grattant le fumier ou chassée de la maison, qui permettent de créer une connivence avec le public des sermons tout en lui transmettant une leçon morale et théologique.

12 Le même Filippe de Ferrare compare implicitement la poule et l'aigle dans le récit suivant : «La poule de l'Empereur Tibère. Un aigle laisse tomber de ses griffes une poule blanche dans les mains de l'empereur Tibère pendant qu'il se promenait dans un jardin. Les sages consultés répondent à Tibère que l'aigle symbolisait l'Empire et la poule tombée dans ses mains la défaite de ses ennemis. » (Vecchio 1998 : n. 62).

13 L'auteur anonyme du Ci nous dit donne une version beaucoup plus religieuse de ce prodige de la blanche géline. "Un empereur et sa femme assis dans un jardin s'inquiétaient de leur succession, parce qu'ils n'avaient pas d'enfant. Alors un aigle qui planait laissa tomber entre eux une poule blanche tenant dans son bec un rameau de laurier. Cet aigle figurait Dieu le Père et la poule blanche la Vierge Marie qui serait notre mère et notre gardienne contre les tromperies du diable avec la vigilance d'une mère poule pour ses 
poussins. » (Blangez 1978-1986, Tubach 1981: n. 2522). Voici un usage particulier de l'image de la mère poule devenue un topos très fréquent.

Les quelques exempla évoquant le coq n'en donnent pas une image aussi positive. L'infatigable prédicateur, Jacques de Vitry compare les mauvais prélats au coq: «Les mauvais prélats sont semblables au coq porté au marché les pieds liés et la tête tournée vers le sol» (Crane 1890: n. 4). Ceci est une manière de rappeler que ces prélats, qui devraient toujours regarder vers le ciel et les réalités supérieures et spirituelles, sont, en réalité, beaucoup plus attirés par les biens terrestres. De plus, ces prélats se révèlent impuissants à mener la moindre action religieuse, comme le souligne la position du coq les pattes liées.

15 Ailleurs, le même prédicateur fustige, dans une fable bien connue et diffusée (Tubach 1981 : n. 3635), la bêtise du coq incapable de reconnaître les véritables richesses. Trouvant dans du fumier une pierre précieuse et une graine pourrie, le coq se précipite sur la graine mais délaisse la pierre précieuse, car elle ne lui est d'aucune utilité (Crane 1890 : n. 54).

Dans un registre plus littéraire, Le Roman de Renart, repris par les prédicateurs, la poule est présentée comme l'éternelle victime du renard.

\section{La poule du Roman de Renart dans la prédication}

17 Toujours selon Jacques de Vitry (Crane $1890:$ n. 297), dans un sermon destiné aux enfants et adolescents, ceux qui confessent leurs péchés pour mieux y retomber font ce que l'on appelle en France la " confession du renard », d'après l'histoire du renard qui confesse ses péchés à un blaireau et lui promet de se corriger. L'instant d'après, il essaie d'attraper une poule, prétextant avoir oublié sa confession. Renart est piégé et ridiculisé par son obsession de dévorer les poules pour les dévorer. Ce récit fut largement repris par ses confrères (TheMA).

Une des fables d'Eudes de Cherinton (Hervieux 1896: IV, n. 50 ; Tubach 1981: n. 2173) rapporte que « Les poules refusent de laisser entrer le renard dans le poulailler. Le renard leur dit qu'il a froid et faim. S'il meurt, Dieu les punira. Prises de pitié, les poules lui ouvrent et sont dévorées. " L'allégorisation nous donne une clé morale et sociale de la fable : «Certains hommes entrent dans les monastères pour bien manger. »

Enfin, le célèbre Bernardin de Sienne reprend ce topos du renard que l'on peut piéger en lui promettant une poule bien dodue à manger, en l'attribuant au loup. Le renard tombe dans un puits et, pour ne pas se noyer, entre dans le seau. Il en sort après avoir convaincu le loup de descendre dans le puits, à l'aide d'un autre seau, pour manger une poule. Cette fable connut un durable succès (Delcorno 1989 : XIII, 1).

Un certain nombre d'exempla s'appuient sur des realia, ou des détails présentés comme des realia, pour en tirer une leçon morale. Ce faisant, ils permettent à l'historien d'approcher, avec toutes les précautions nécessaires, une certaine réalité sociale.

\section{Exempla et realia}

21 La poule apparait, dans certains milieux, comme un bien ordinaire plus ou moins valorisé chez les laïcs selon leur statut social. 

parmi les choses qu'un voleur peut cacher sous son surcot : « Le pacte du voleur de petites choses. Le diable trompe habilement ceux qui tombent dans ses filets. Un diable apparut à un pauvre garçon qui n'avait pas trop de courage. Il lui dit de ne pas hésiter à voler des petites choses comme couteaux, chausses, bonnets, poules, canes et canards, hanaps d'argent, toutes choses qu'on peut cacher sous son surcot : " et ainsi tu deviendras vite riche. " " La suite de l'histoire est fatale pour le voleur qui finit par voler un bœuf et se faire condamner par la justice (Blangez 1979-1986 : n. 202). compilations. Serait-ce une forgerie de l'auteur du Ci nous dit qui met ainsi en scène le proverbe «Qui vole un œuf, vole un bœuf » (non repéré dans Morawski 1925). Cela est bien possible. Ce procédé convient sans doute également à ce court exemplum proposé par Bernardin de Sienne dans un sermon à Sienne en 1425 : «Une femme ne trouvant plus une de ses poules la cherche longtemps jusqu'à ce qu'elle la trouve ». D'où la leçon : si on passe tant de temps pour retrouver un bien limité, d'autant plus de temps sera employé pour conserver son âme. Sermon "Celui-ci est le sermon que Frère Bernardin fit sur l'âme, et montre en quelle grande dignité elle est créée, et combien Dieu l'estime » (Cannarozzi 1958 : I, 96).

Dans ce dernier cas, la poule apparaît comme le type de bien de peu d'importance, aux yeux du prédicateur, mais pas de cette femme, sans doute d'un milieu modeste. De plus, on peut se demander s'il n'y a pas derrière ce récit, trivial en apparence, une allusion à la parabole du bon berger.

De la même manière, Jean Gobi rapporte dans la rubrique de la Scala coeli consacrée aux prêtres (et essentiellement à leurs défauts) : « Une bonne petite vieille gravement malade fait venir chez elle le curé de sa paroisse pour faire son testament. Elle lui lègue sa poule, que le curé fait chercher dès le lendemain, pour la manger. La petite vieille guérit, et accuse le prêtre d'être pire que le diable.» (Polo de Beaulieu 1991 : n. 925, Tubach 1981 : n. 2423).

ans le Ci nous dit (Blangez 1979-1988: n. 270, Tubach $1981:$ n. 4989, 5267), on découvre qu'un des célèbres jugements de Trajan concernait la mort d'une poule :

"Trajan fait justice à une veuve. Le fils d'un empereur de Rome lâcha son autour, qui tua la poule d'une pauvre femme. Le fils de cette femme tua l'autour. Le fils de l'empereur tua le garçon. La femme dit à l'empereur : - " Sire, faites-moi justice ! Ce jeune homme m'a tué ma poule et mon fils." Il répondit:- "Je te ferai justice quand je reviendrai. - Ah, sire, dit-elle, et si vous ne revenez pas, qui me fera justice ? - Femme, dit l'empereur, je suis obligé de te faire justice : Je te ferai servir comme une impératrice pourvu que mon fils ne meure pas; mais si tu préfères il mourra ". La femme accepta et il la fit installer richement. Quand l'empereur fut mort, on grava ses plus beaux jugements sur son tombeau. Et depuis il fut sauvé grâce à la prière de saint Grégoire qui avait lu ses beaux jugements sur son tombeau ».

Ce récit met en scène un enchaînement terrible de vengeances à partir de la mort d'une poule, modeste bien aux yeux de l'empereur, mais pas à ceux de sa pauvre propriétaire.

Dans l'optique cléricale, s'occuper des poules et de bestiaux est un signe d'humilité, de la part d'un ancien noble devenu moine, comme l'explique le dominicain Étienne de Bourbon : « Un comte de Poitiers, devenu cistercien, demanda à s'occuper des poules, des moutons et des porcs; il s'émerveillait devant ces animaux, créatures de Dieu. » (Berlioz (Ed.) 2006 : n. 1292, Tubach $1981:$ n. 258).

Revue d'ethnoécologie, 12 | 2017 

l'atteste ce récit qui connut un immense succès jusqu'à devenir un mystère à la fin du Moyen Âge, mais avec un crapaud à la place du serpent (Berlioz 1990). Une des premières versions apparaît dans un sermon du cistercien Césaire de Heisterbach (Coppenstein 1615 : 141AB, Hilka 1933 : t. I, p. 94 : n. 77, Strange 1851 : VI, 22, Tubach $1981:$ n. 4883) : Henri, un chevalier de Moselle, persuada sa mère veuve de lui céder ses possessions ; ceci fait, il la chassa de la maison. Un soir, quand la famille d'Henri était à table en train de manger un poulet, la mère désespérée frappa à la porte. Henri ordonna à un domestique de cacher une écuelle avec de la nourriture, jusqu'à ce que "le Diable soit parti ». Une fois la mère partie, le domestique retrouva dans l'écuelle un serpent au lieu du poulet. Henri, qui voulait voir lui-même le serpent, se pencha sur l'écuelle, mais le serpent se jeta à son cou tant et si bien qu'on ne réussit pas à le défaire du serpent : si on le touchait, le serpent serrait le cou de sa victime. C'est ainsi que Henri fut obligé de vivre avec le serpent et de le nourrir car, affamé, il menaçait toujours de l'étrangler. Dans l'espoir d'obtenir sa libération, Henri, accompagné de sa mère, était montré en spectacle de sanctuaire en sanctuaire. divine se manifeste dans ce récit, où la poule, en tant que nourriture carnée, est interdite à des religieux durant le Carême :

«Comment furent punis le moine et le clerc qui voulaient manger une poule pendant la nuit des Cendres. Après avoir bu du vin la nuit des Cendres, un moine et un clerc décident de manger une poule; ils trouvent un crapaud vivant dans ses entrailles et comprennent alors que c'est le diable.» (Vecchio 1998: n. 69, Tubach 1981 : n. 4878).

Filippe de Ferrare insiste sur le fait que ce désir de consommer un aliment interdit lors de la nuit des Cendres, fête liturgique importante marquant l'entrée en Carême, est une tentation diabolique. La sainteté de François d'Assise qui s'abstenait toute l'année de viande, apparaît d'autant plus remarquable dans cet exemple rapporté dans le Ci nous dit : "Un jour saint François fut malade. Une fois guéri, il se mit une corde au cou et alla au gibet. Les gens le suivirent, croyant qu'il perdait la raison. Quand tous furent assis autour de lui, il leur dit: "Bonnes gens, je vais vous dire pourquoi je suis venu ici. J'ai l'habitude de m'abstenir de viande, vous le savez tous. Pour ne pas devenir escroc et hypocrite devant Dieu, je vous annonce à tous que mes compagnons m'ont fait manger de la poule pendant ma maladie. Je ne veux pas mener une vie qui ne soit connue de tous. On voit ici que nous devons tous être meilleurs en nous-mêmes que nous ne paraissons à l'extérieur. Nous ne devons pas être honorés pour notre apparence, mais pour les mœurs et les vertus qui doivent se trouver en nous. Car ce n'est pas le costume qui doit faire honneur au maître, mais c'est au maître à faire honneur au costume. » (Blangez 1979-1986 : n. 735). Ce récit nous indique un usage médicinal de la poule ${ }^{12}$. Il a pu être inspiré par la première Vie de François de Thomas de Celano (1C, 52 : Dalarun 2010 : 530).

À l'inverse de cette continence des religieux, manger de la poule semble bien une nourriture indigne d'un roi, comme le raconte Filippe de Ferrare dans un récit sans source connue : 
«Le roi d'Angleterre et les cornes de cerf. Un roi d'Angleterre se perdit en forêt, lors d'une battue de chasse, il fut reçu par un paysan qui lui offrit au dîner des poules. Le roi n'étant pas reconnu demanda de la viande de cerf et le paysan la lui prépara sous la promesse de ne pas le dénoncer au roi pour avoir violé les droits royaux de chasse. Le jour suivant, le paysan guida son hôte en ville et le roi, désormais à distance d'un mille de chez lui, sonna du cor, le cor du diable, et ses soldats arrivèrent rapidement. Ayant compris qui était son hôte, le paysan prit peur parce qu'il lui avait offert de la viande de cerf, mais le roi le rassura et l'invita à déjeuner et lui promit de lui donner ce qu'il voulait. Le paysan demanda une parcelle de terre à cultiver pour lui et sa famille. » (Vecchio $1998:$ n. 209).

Au détour de l'histoire du fils du comte de Crépy, devenu mendiant pour le Christ, on apprend qu'une pauvre veuve lui offre en aumône une croûte de pain noir et un fragment de tuile dans lequel buvaient les poules. Voici un détail dont on peut penser qu'il s'agit d'un détail authentique de la vie quotidienne, rapporté par Étienne de Bourbon dans un récit exemplaire qui connut un grand succès (Berlioz (Ed.) 2002: n. 346, Tubach 1981 : n. 4880).

Les exempla nous font également découvrir tout un savoir naturaliste moralisé sur la poule, issu des bestiaires.

\section{Savoir naturaliste moralisé sur la poule}

Les bestiaires, et particulièrement les encyclopédies produites en abondance à partir du $\mathrm{XIII}^{\mathrm{e}}$ siècle, sont toutes explicitement liées à un projet pastoral (leitmotiv de leurs prologues) : la connaissance naturaliste est mise au service de la prédication, devenue priorité de la papauté en lutte contre les hérésies et les superstitions depuis le concile de Latran IV (1215).

Thomas de Cantimpré décrit et moralise une caractéristique curieuse de la poule :

«Un fait admirable tiré de Pline. Dans le livre De la nature des choses, dont Pline est l'auteur, il est dit que la poule ne peut être piquée par un serpent le jour où elle a pondu un œuf. Qu'est-ce que l'œuf, espoir d'un poussin, si ce n'est le fruit de la bonne action par lequel est désigné l'espoir d'une vie éternelle?».

En réalité, il convient de distinguer deux étages de sources : le Liber de natura rerum $(5,58$, 50) de Thomas de Cantimpré (Boese 1973 : 207), qui s'inspire explicitement de l'Histoire naturelle (livre 29) de Pline l'Ancien. La moralisation se focalise sur l'œuf et le poussin, oublie la poule et introduit une perspective eschatologique qui correspond bien à la définition de Jacques Le Goff de l'exemplum homilétique, qui transmet une leçon salutaire, au sens le plus fort de salut dans l'au-delà (Le Goff et al. 1982-1996 : 37-38).

Jacques de Voragine utilise dans ses sermons de Carême les traits des poussins pour donner une leçon sur les bons chrétiens qui suivent le Christ comme les poussins suivent leur mère poule. Le thème de cette partie finale du sermon est la lamentation du Christ sur Jérusalem : "Que de fois j'ai voulu rassembler tes enfants comme une poule rassemble ses poussins sous ses ailes, et vous n'avez pas voulu !» (Mt 23).

Peut-être tirée d'une observation personnelle, Étienne de Bourbon rappelle que les poules veulent moins venir en août à la maison de leur maître qu'en hiver où elles trouvent peu de choses à manger (Berlioz $2006:$ n. 1307). De même, les recueils de distinctiones bibliques de Nicolas de Biard (1275-1285) et de Nicolas de Gorran O. P. (†1295) bien connus des prédicateurs, utilisent une locution proverbiale assez proche de l'exemplum: «sourd 
comme une poule au mois d'août» (Berlioz \& Polo de Beaulieu 1999: 181). En bon prédicateur, Étienne de Bourbon tonne contre cette ingratitude des volailles envers leur maître qui les nourrit, revenant à la leçon initiale qui introduit la comparaison : «Parfois ceux qui sont le mieux nourris sont les plus ingrats envers leur bienfaiteur ».

De la même manière, selon le compilateur du Ci nous dit, le coq maigre parce qu'il laisse à ses poules le bon grain est-il comparé au prédicateur qui ne met pas en pratique ce qu'il prêche : il mène les gens en paradis et se mène lui-même en enfer (Blangez 1979-1986: n. 297).

\section{La poule et le poulet symboles de la Vierge, du Christ et de Dieu}

Quelques prédicateurs n'hésitent pas à assimiler la Vierge, le Christ et même Dieu à une poule ou un poulet. Dans ses sermons sur la Vierge, Jacques de Voragine (Clutius 1760) développe longuement une comparaison entre la Vierge et la poule : « Les poules ayant des poussins sont comme Marie.»

La poule, plus que tout autre animal possède quatre traits que Marie a assumés: la compassion, la sollicitude, la magnanimité et le soin assidu pour couver ses œufs. Elle porte toute la compassion car elle compatit tant pour les poussins petits et malades qu'elle semble elle-même souffrir en même temps qu'eux. Elle montre cette compassion dans ses plumes ou ses ailes, car elle devient totalement hérissée (hispida), et dans sa voix qui devient rauque.

«Elle porte aussi toute la sollicitude et cela de deux manières : pour nourrir et pour surveiller <ses poussins>, car elle cherche partout des grains, et quand elle en trouve, elle fait venir là les poussins en criant à plein gosier. Deuxièmement dans la surveillance <des poussins> qu'elle garde sous ses ailes et protège des attaques du milan, comme Dieu (Mt $23: 37$ ).

Elle porte toute la magnanimité car alors que les poules et les autres oiseaux fuient naturellement les hommes qui les suivent, la poule cependant se retourne contre ces hommes et les chasse de son bec et par ses cris.

Elle porte aussi toute la diligence pour couver les œufs, elle oublie <de s'occuper d'ellemême> et les couve sans cesse. Pour toutes ces raisons, les poules peuvent être comparées à la Vierge Marie. »

Puis le prédicateur reprend toutes ces qualités et montre comment la Vierge les a assumées durant sa vie et comment elle les assume encore à l'égard des fidèles, images de ses poussins.

On trouve une assimilation du Christ à une volaille poussée dans ses plus extrêmes conséquences dans un curieux exemplum, resté un hapax, de la Table des exemples, au chapitre sur la Passion du Christ (Welter 1927 : n. 216) :

«Le Christ placé sur la croix était comme un poulet grillé, car il fut d'abord déplumé et dépouillé, puis fixé sur la broche de la croix à l'aide de clous en fer et ensuite érigé en hauteur, placé face à l'ardeur du soleil. Et de même que pour vérifier si une volaille est bien cuite, on plante un couteau affuté dans sa poitrine : si aucune goutte de sang ne sort, mais de la graisse blanche, on dit que le poulet est cuit. On lui fit la même chose : en effet, une lance transperça son flanc, et alors une graisse blanche en sortit, ensuite disposée comme une viande cuite la graisse de la 
charité et de la miséricorde ne lui fit pas défaut, elle qui s'écoulait de tous les membres de son corps $[. .]$.$» .$

$$
\text { se reporter à un exemplum du même chapitre (Welter (Ed.) } 1927 \text { : n. 217a) : }
$$

« De même qu'un homme vaincu en duel ou à la guerre refusera de demeurer dans

la maison dans laquelle est peinte sa défaite, de même le diable ne restera pas dans

l'âme portant la peinture de la Passion du Christ grâce au travail de la mémoire. »

51 Ce contexte narratif insiste donc sur la nécessité de garder toujours en mémoire la Passion du Christ pour faire son salut. Les larmes peuvent être la preuve de cette dévotion aux souffrances du Christ (Nagy-Zamboni 2000). Ce modèle de piété offert aux simples laïcs revient de façon récurrente dans les sermons ad populum. C'est dans ce cadre que s'insère l'exemplum reposant sur une comparaison terme à terme entre la cuisson du poulet et la Passion du Christ. un terme pour évoquer le Christ aux outrages (mis à nu, moqué, etc.) évoqué par les participes deplumatus et expoliatus, puis la mise en croix, assimilée à l'installation de la volaille sur la broche, l'élévation de la croix (in altum levatus) comparée à la cuisson (ici au feu du soleil), et enfin le percement du côté du Christ, ramené ici à un simple test de cuisson.

On ne peut imaginer de comparaison plus triviale et humiliante pour le Christ, objet passif de préparatifs en apparence purement culinaires. Mais la fin de l'exemplum donne la clef théologique de cette métaphore pour le moins étonnante : la graisse blanche qui s'écoule de tous les membres du corps du Christ représente sa miséricorde et sa charité. Cette cuisson banale est en réalité une image de la rédemption du genre humain.

Ce corps saint entre tous, qui répand une graisse blanche miraculeuse, rappelle certains sarcophages de saints qui émettaient en permanence ou à la date de leur fête une huile miraculeuse, pour le plus grand profit des pèlerins (Sigal 1985).

Le Franciscain anonyme semble particulièrement affectionner les métaphores culinaires pour évoquer la Passion du Christ et l'eucharistie. La rubrique qui est consacrée à cette dernière est intitulée "Pain de l'eucharistie », ce qui est un hapax dans les recueils à classement logique alphabétique (en général on trouve les intitulés eucharistie, sacrement de l'eucharistie, communion). Ici, l'eucharistie est décrite comme pain et vin, corps et sang du Christ, chair sortie du lardier de la sainte Vierge et préparée au sel des souffrances sur le gibet de la croix (Welter (Ed.) 1927 : n. 219).

L'exemplum traduit ci-dessus renvoie à une pratique largement attestée : la cuisson à la broche, plutôt réservée aux milieux aisés, si l'on en croit l'archéologie et les inventaires après décès (Piponnier 1996 : 528, Riera-Melis 1996 : 405-407). Cette volonté d'expliquer le plus concrètement possible les mystères de la foi et les pratiques religieuses sont peutêtre une caractéristique des recueils d'exempla franciscains. D'ailleurs, l'auteur de la Tabula exemplorum revendique clairement cette approche concrète et se moque de ceux qui voudraient raisonner, poser des questions, évaluer des arguments concernant l'eucharistie et la transsubstantiation, affirmée comme dogme au dernier concile de Latran (Latran IV, 1215). 

(Hervieux 1896: IV, n. 34) compare la poule qui défend ses petits contre le milan, en les recouvrant de son aile, à Dieu. Mais l'un de ses poussins est attiré par un ver et va le picorer. Le milan l'emporte. Le milan représente le diable.

comparaison est reprise dans la traduction espagnole des fables d'Eudes de Cherinton (Gatos, 36) et dans les Gesta Romanorum au succès durable (Oesterley 1872: n. 51). Dans le Ci nous dit, la poule est comparée au Christ: «En étendant ses ailes <le Christ> a vaincu le diable, comme la mère poule qui s'élève en l'air contre le milan pour protéger ses poussins. De même Jésus s'est élevé en l'air, les ailes étendues, pour nous protéger contre les diables. » (Blangez 1979-1986 : n. 82, Tubach 1981 : n. 1005).

59 dépasse l'image des ailes de la poule protectrice. Ces deux allusions aux ailes protectrices de la poule renvoient directement à l'Évangile de Matthieu (Mt $23: 37$ ), reprises par Luc (Lc 13 : 34-35) dans la lamentation de Jésus sur Jérusalem : « Jérusalem, Jérusalem, toi qui tues les prophètes et lapides ceux qui te sont envoyés, que de fois j'ai voulu rassembler tes enfants comme une poule rassemble ses poussins sous ses ailes, et vous n'avez pas voulu !» Cette citation apparaissait déjà dans un des sermons de Jacques de Voragine.

édicateur se réclame d'un Livre des propriétés des oiseaux (que nous n'avons pas encore identifié) pour expliquer que parfois la poule couve des œufs de canard et de paon au milieu de ses propres œufs. Quand les œufs éclosent, les véritables poussins suivent leur mère, les canards se jettent à l'eau et les paons fiers de leur beauté s'éloignent. De la même manière, Dieu, comme un bon pasteur et une bonne poule, réunit les bons et les méchants dans une même foi, et les soigne par ses nombreux bienfaits. Alors certains le suivent par imitation des bonnes œuvres, comme les humbles et les miséricordieux, d'autres se plongent dans les eaux des plaisirs comme les voluptueux et enfin d'autres s'éloignent en se glorifiant de leurs richesses : ce sont les orgueilleux et les avares. Alors Dieu leur dit à tous «Suivez moi » (Clutius $1760: 135 \mathrm{a}-\mathrm{b}$ 2). On retrouve cette image très commune dans les sermons inédits du frère augustin Simon Cupersi vers 1460 (Berlioz \& Polo de Beaulieu 1999 : 165).

61 À l'inverse de cette image maternelle, le coq cuit peut devenir l'instrument de la terrible vengeance du Christ contre des pécheurs coupables de blasphème. Depuis les lettres de Pierre Damien à la fin du XI ${ }^{e}$ siècle (Reindel 1989 : lettre 119 374-375), ce récit est passé dans la littérature exemplaire cistercienne (Hélinand de Froidmont, Chronicon, a. 1078; PL, 212, col. 974C-975A; Berlioz \& Polo de Beaulieu 2012 : n. 666), puis dominicaine (Polo de Beaulieu 1991: n. 160). Elle a connu un très grand succès (Tubach 1981: n. 1130). Voici la version de Filippe de Ferrare :

«Le coq ressuscité. À Bologne, deux clercs tuent un coq et le coupent en petits morceaux pour le manger. L'un des deux clercs dit à l'autre pour plaisanter que même saint Pierre ne réussirait pas à remettre ensemble tout le coq ; l'autre répond que Jésus Christ en personne ne pourrait réussir non plus. Le coq se lève, chante et, en battant des ailes, projette toute la sauce sur les deux clercs blasphémateurs qui attrapèrent ainsi la lèpre. Cette maladie fut également infligée à tous leurs descendants. » (Vecchio $1998:$ : n. 74).

D'une manière implicite, le coq ressuscité représente le Christ ou Dieu et porte sa vengeance contre les blasphémateurs. Vengeance particulièrement terrible dans cette version, car elle s'étend à toute la descendance des pécheurs, bien au-delà donc de la responsabilité individuelle.

Revue d'ethnoécologie, 12 | 2017 
$63 \mathrm{Au}$ fil des sermons et des exempla, en fonction des sources utilisées par les prédicateurs et selon la thématique religieuse à illustrer, la poule est apparue comme le symbole de l'animal ordinaire, familier, soit passif (victime de Renart, son sang servant de médicament), soit actif dans le rôle de la mère poule prête à tous les sacrifices et à toutes les audaces pour protéger ses poussins. En ce sens, elle peut soutenir la comparaison avec la Vierge Marie, le Christ et même Dieu.

Ce panorama aussi bref que contrasté des représentations de la poule (et par extension du poulet) dans les exempla et les sermons du Moyen Âge appelle quelques remarques finales. Les exempla peuvent laisser filtrer des détails de la vie quotidienne précieux pour l'historien (la tuile ébréchée servant de récipient pour la poule). Il convient de les repérer et de les analyser avec précaution pour les distinguer des topoï.

Leur valeur informative est importante sur la vulgarisation d'un savoir naturaliste aux sources multiples bien identifiées, mais qui s'enrichit de gloses issues de l'exégèse et des traités de théologie, sans oublier les reconfigurations opérées par le discours pastoral.

Ces représentations sont à penser en réseau, dans le cadre d'une hiérarchisation du règne animal, dans un réseau d'allégories qui se figent parfois en stéréotypes.

De ce fait, pour comprendre une représentation textuelle ou figurée de la poule au Moyen Âge, il conviendra de tenir compte de ces couches successives de significations spirituelles et morales qui ont sédimenté au fil des siècles de compilations en réécritures, sans que la créativité propre à la performance du prédicateur ne nous parvienne intégralement malgré les notes (reportationes) plus ou moins fidèles d'auditeurs clercs ou laïques.

\section{BIBLIOGRAPHIE}

AASS : Acta Sanctorum on line http://www.bollandistes.org/publications.php?pg=acta-sanctorum Amadori S. 1996 - Una nuova fonte sacchettiana: il Liber de introductione loquendi di Filippo da Ferrara. Lettere Italiane XLVIII : 420-436.

Berlioz J. 1990 - L'homme au crapaud. Genèse d'un exemplum médiéval. In Tradition et Histoire dans la culture populaire. Rencontres autour de l'œuvre de Jean-Michel Guilcher, Grenoble, musée Dauphinois (20-21 janvier 1989). Grenoble, Centre alpin et rhodanien d'ethnologie : 169-203. (Documents d'ethnologie régionale ; 1 ).

Berlioz J. 2002 - Le bel oiseau ambigu. Gryphe. Revue de la Bibliothèque de Lyon 5, $2^{\mathrm{e}}$ semestre : 22-27.

Berlioz J. \& Jean-Luc Eichenlaub (Ed.) 2002 - Stephani de Borbone Tractatus de diversis materiis predicabilibus. Prologus. Prima pars de dono timoris. Turnhout, Brepols, XCI-638 p. (Corpus Christianorum. Continuatio Mediaeualis ; 124)

Berlioz J. (Ed.) 2006 - Stephani de Borbone Tractatus de diversis materiis predicabilibus. Tertia pars de dono scientie. Turnhout, Brepols, XXVIII-684 p. (Corpus Christianorum. Continuatio Mediaeualis ; 124B). 
Berlioz J. \& Cordonnier R. 2004 - Le convers et les oiseaux. Monde animal, morale et milieu monastique : le De avibus d'Hugues de Fouilloy (XII ${ }^{\mathrm{e}}$ siècle). In : Homme animal. Histoires d'un face à face. Strasbourg-Paris, Éditions des musées de Strasbourg/Adam Biro : 73-81.

Berlioz J. Ogilvie-David D. \& Ribaucourt C. (Ed.) 2015 - Stephani de Borbone Tractatus de diversis materiis predicabilibus: Secunda pars de dono pietatis. Turnhout, Brepols, XXVIII-690 p. (Corpus Christianorum. Continuatio Mediaeualis, 124A).

Berlioz J. \& Polo de Beaulieu M. A. (Ed.) 1999 - L'animal exemplaire au Moyen Âge, $V^{e}-X V^{e}$ siècles. Rennes, PUR, 329 p. (Histoire).

Berlioz J. \& Polo de Beaulieu M.A. (Ed.) 2012 - Collectio exemplorum cisterciensis in codice Parisiensi 15912 asseruata. Turnhout, Brepols, xlii-632 p. (Corpus Christianorum Continuatio Mediaevalis ; 243).

Bertini Guidetti S. 1998 - I sermones di Iacopo da Varazze: Il potere delle immagini nel Duecento. Florence, SISMEL, Edizioni del Galluzzo, x-174 p.

BHL - Bibliotheca Hagiographica Latina on line : http://bhlms.fltr.ucl.ac.be

Blangez G. 1979-1986 - Ci nous dit. Recueil d'exemples moraux. Paris, SATF, 2 vol., t. 1, 1979, cxxix-331 p. ; t. 2, 1986, 478 p.

Boese H. (Ed.) 1973 - Thomas de Cantimpré, Liber de natura rerum. Berlin-New York, W. de Gruyter , xi-431 p.

Boivin J.-M. et al. (Ed.) 2011 - Les fables avant La Fontaine, colloque international de Paris-La Sorbonne. Genève, Droz, 503 p. (Publications romanes et françaises ; 203).

Boureau A. 1999 - L'animal dans la pensée scolastique. In : Berlioz J. \& Polo de Beaulieu M.A. (Ed.), L'Animal exemplaire au Moyen Âge, $V^{e}-X V^{e}$ siècles. Rennes, PUR : 99-109. (Histoire).

Boglioni P. 1999 - Les animaux dans l'hagiographie monastique. In : Berlioz J. \& Polo de Beaulieu M.A. (Ed.) - L'animal exemplaire au Moyen Âge, $V^{e}-X V^{e}$ siècles. Rennes, PUR : 51-80. (Histoire).

Cannarozzi P. C. 1934 - Bernardino da Siena, Le Prediche volgari. Pistoia, Pacinotti, 2 vol., xlvi-463 p.-504 p.

Cannarozzi P. C. 1958 - Predicazione del 1425 in Siena. Florence, Rinaldi, 2 vol. xl-300 p., 390 p.

Clutius (Ed.) 1760 - Jacobus de Voragine, Sermones aurei. Sermones quadragesimales, Paris, 220 p.

Coppenstein J.A. 1615 - Fasciculus moralitatis venerabilis fr. Caesarii Heisterbacensis monachi... Homilias de infantia Servatoris Iesu Christi. Cologne, 14-159 p.

Crane T. F. 1890 - The Exempla or Illustrative Stories from the Sermones vulgares of Jacques de Vitry. Londres, Nutt, The Folk-Lore-Society Publications xxvi, cxvi-303 p.

Creytens R. 1946 - Le manuel de conversation de Philippe de Ferrare O. P. († 1350). Archivum Fratrum Praedicatorum $16: 107-135$.

Dalarun J. (Ed.) 2010 - François d'Assise. Écrits, vies, témoignages. Paris, Cerf/Éditions franciscaines, iii-3418 p. (Sources franciscaines).

Delcorno C. 1989 - S. Bernardino da Siena, Prediche volgari sul Campo di Siena 1427. Milan, Rusconi, 2 vol., $1422 \mathrm{p}$.

Heck C. 2012 - Le Ci nous dit. L'image médiévale et la culture des laïcs au XIV siècle : les enluminures du manuscrit de Chantilly. Turnhout, Brepols, 358 p.

Hélinand de Froidmont - Helinandus Frigidimontis, Chronicon. Patrologia Latina, t. 212, 1855, colonnes 9-1087, spécialement colonnes 771-1082. 
Hervieux L. 1896 - Les fabulistes latins depuis le siècle d'Auguste jusqu'à la fin du Moyen Âge, t. IV, Eudes de Cheriton et ses dérivés. Paris, Firmin-Didot, viii-483 p.

Hilka A.-B. (Ed.) 1933 - Die Wundergeschichte des Caesarius von Heisterbach, t. 1. Bonn, 234 p. (Publikationen der Gesellschaft für Rheinische Geschichtskunde ; 43).

Le Goff J., Bremond C. \& Schmitt J.-C.1982-1996 - L'Exemplum. Turnhout, Brepols, 166 p. (Typologie des sources ; 40).

Longère J. 1982 - Odon de Cherinton. In Dictionnaire de Spiritualité, 11 : col. 618-620.

Longère J. (Ed.) 2013 - Iacobus de Vitriaco, Sermones uulgares uel ad status. Turnhout, Brepols, cccm-255, cxiv-790 p.

McGuire B.P. 2004 - Cistercian Storytelling - A living Tradition: Surprises in the World of Research. Cistercian Studies Quaterly 39 : 281-309.

Maggioni G.P. (Ed.) 2005 - Iacopo da Varazze, Sermones Quadragesimales, Edizione critica. Florence, SISMEL, Ed. del Galluzzo, lxiii-613 p. (Edizione nazionale dei testi mediolatini ; 13, serie I, 8)

Morawski J. 1925 - Proverbes français antérieurs au Xve siècle. Paris, Champion, xxiii-147 p. (Les classiques français du Moyen Âge ; 47).

Nagy-Zamboni P. 2000 - Le Don des larmes au Moyen Âge. Un instrument spirituel en quête d'institution ( $V^{e}$-XIII ${ }^{e}$ siècle). Paris, Albin Michel, 448 p. (Histoire).

Oesterley H. (Ed.) 1872 - Gesta Romanorum. Berlin, Weidmann, viii-756 p.

Piponnier F. 1996 - Du feu à la table : archéologie de l'équipement de bouche à la fin du Moyen Âge. In : Montanari M. et Flandrin J.-L. (Ed.) Histoire de l'alimentation. Paris, Fayard, 915 p.-[16] p. de pl. : 525-537.

Platelle H. 1997 - Les Exemples du Livre des abeilles. Présentation, traduction et commentaire. Turnhout, Brepols, 383 p. (Miroir du Moyen Âge).

Polo de Beaulieu M.A. 1991 - La Scala cœli de Jean Gobi, présentation et édition. Paris, Éditions du CNRS, 766 p. (Sources d'histoire médiévale/IRHT).

Polo de Beaulieu M.A. 1999 - Éducation, prédication et cultures au XIVe siècle. Essais sur Jean Gobi le Jeune († 1350). Lyon, PUL, $237 \mathrm{p}$.

Polo de Beaulieu M.A. 2010 - De l'exemplum monastique à l'exemplum mendiant : continuités et ruptures. In : Forster R. \& Günthart R. (Ed.), Didaktisches Erzählen. Formen literarischer Belehrung in Orient und Okzident. Francfort-sur-le-Main-Berlin-Berne, Bruxelles-New York-Oxford-Vienne, Peter Lang Verlag : 55-84.

Polo de Beaulieu M.A., Berlioz J. \& Collomb P. (Ed.) 2010 - Le Tonnerre des exemples. Exempla et médiation culturelle dans l'Occident médiéval. Rennes, Presses universitaires de Rennes, $423 \mathrm{p}$. (Histoire).

Reindel K. (Ed.) 1983-1993 - Die Briefe des Petrus Damiani. MGH-Briefe des deutschen Kaiserzeit IV, 4 vol., Munich, Monumenta Germaniae Historica, t. 3, 557 p.

Riera-Melis A. 1996 - Société féodale et alimentation (XII ${ }^{\mathrm{e}}-\mathrm{XIII}{ }^{\mathrm{e}}$ siècle). In Montanari M. \& Flandrin J.-L. (Ed.), Histoire de l'alimentation. Paris, Fayard : 397-418.

Sigal P.-A. 1985 - L'Homme et le miracle dans la France médiévale. Paris, Le Cerf, 349 p.

Smirnova V., Polo de Beaulieu M.A. \& Berlioz J. 2015 - The Art of Cistercian: Persuasion in the Middle Ages and Beyond. Caesarius of Heisterbach's Dialogue on Miracles and Its Reception. Boston-Leyde, Brill, $295 \mathrm{p}$. 
Strange 1851 (Ed.) - Caesarius Heisterbacensis, Dialogus miraculorum. Cologne, Bonn, Bruxelles, J.M. Heberle, 2 vol., t. 1, vi-406 p. et t. 2, 381 p.

TheMA - Thesaurus exemplorum Medii Aevi on line http://gahom.huma-num.fr/thema/

Thomas de Cantimpré 1627 - Bonum universale de apibus. Douai, ex pyp. Baltazaris Belleri, 41 p. 1., $594,176,[54] \mathrm{p}$.

Tubach F.C. 1981 - Index exemplorum. A Handbook of Medieval religious tales. Helsinki, Helsingin Liikekirjapaino Oy, 530 p. (Fellow Folklore Communications ; 204).

Vecchio S. 1998 - Il Liber de introductione loquendi di Filippo da Ferrara. In : I Castelli di Yale, III. Padoue, Il Poligrafo impr. : 131-165.

Welter J.-T. (1927) 1973 - L'exemplum dans la littérature religieuse et didactique du Moyen Âge. ParisToulouse, Slatkine, $562 \mathrm{p}$.

Welter J.-T. (Ed.) (1927) 1973 - Tabula exemplorum secundum ordinem alphabeti. Paris-Toulouse, Slatkine, $151 \mathrm{p}$.

Zinc M. 1976 - La prédication en langue romane avant 1300. Paris, Honoré Champion, 581 p. (Nouvelle Bibliothèque du Moyen Âge ; 4).

\section{NOTES}

1. Après douze ans passés dans les États latins d'Orient, comme évêque de Saint-Jean d'Acre de 1216 à 1228, Jacques de Vitry revient en Occident où il compose et prononce des centaines de sermons jusqu'à sa mort (1240), dont les sermons pour le peuple ou par statut social qui font la part belle aux exempla hauts en couleurs émaillés de proverbes, refrains et expressions en langue vulgaire.

2. Originaire du Kent, Eudes de Cheriton († 1246) a été formé à Paris, puis il a voyagé en France et en Espagne. Il mourut en 1246 et fut inhumé dans la cathédrale de Rochester. Il a laissé une série de sermons et des traités théologiques. Ses Fables et Paraboles (Fabulae et Parabolae) constituent son œuvre la plus diffusée, rapidement intégrées dans les recueils d'exempla et traduites en français et en espagnol (Libro de los Gatos).

3. Le cistercien Césaire de Heisterbach est l'auteur d'un recueil d'exempla sous forme dialoguée entre un novice et un moine expérimenté, le Dialogue des miracles (1217-1223), qui déploie en douze livres un itinéraire spirituel. Il a réemployé nombre de ses exempla dans ses sermons. Loin de s'en tenir au "storytelling monastique », Césaire de Heisterbach a profité de ses voyages pour collecter de nombreux récits parmi les laïcs. Les animaux y sont parfois représentés mais jamais sous la forme de la fable ou du proverbe (Polo de Beaulieu 2010).

4. Le dominicain Jacques de Voragine, archevêque de Gênes (1292-1298), a laissé une Légende dorée , qui a été le best-seller du Moyen Âge, avec un millier de manuscrits latins conservés. Par analogie, l'éditeur (Clutius 1760) a regroupé ses 700 sermons conservés sous le titre de Sermons dorés (Sermones aurei). Ils comprennent quatre collections: les sermons de Carême ( Quadragesimales) édités par G. P. Maggioni (Maggioni éd. 2005), les sermons sur les saints (de sanctis), de la Vierge (Mariale) et les sermons du temps (de tempore). Le succès des sermons est impressionnant. Ces sermons ont été écrits soit après 1267, soit après 1277.

5. Étienne de Bourbon fut formé chez les dominicains de Paris avant de revenir à Lyon. Il remplit de nombreuses missions de prédication puis d'inquisition à partir de 1236 environ. Vers 1250, Étienne de Bourbon se retira dans le couvent de Lyon pour y rédiger son Traité des diverses matières à prêcher ou Livre des Sept dons du Saint Esprit, laissé inachevé par sa mort en 1261. Ce vaste 
recueil se compose d'environ 3000 exempla et connut un succès durable, et nombre de ses confrères y puisèrent leur inspiration.

6. D'abord formé à l'abbaye des chanoines réguliers de Cantimpré près de Cambrai, Thomas entra ensuite chez les Frères prêcheurs au couvent de Louvain, qui l'envoyèrent étudier auprès d'Albert le Grand, sans doute à Cologne, à Paris au couvent Saint-Jacques. De retour à Louvain en 1246, il se consacra surtout à la prédication et à l'écriture jusqu'à sa mort vers $1270-1272$. Il a laissé des Vies de mystiques flamandes, mais il nous intéresse ici pour son Liber de natura rerum dont la première rédaction se place avant 1244 (Boese 1973) auquel il fit nombre d'emprunts pour son recueil d'exempla intitulé : Le Bien universel selon les abeilles (Bonum universale de apibus) composé entre 1256 et 1263 . Les deux ouvrages furent couronnés de succès.

7. La Scala coeli a été composée par un dominicain lecteur du couvent royal de Saint-Maximin en Provence, Jean Gobi le Jeune (Polo de Beaulieu 1991). C'est un volumineux recueil d'un millier d' exempla répartis dans 122 rubriques rangées dans l'ordre alphabétique, de Abstinentia à Usura, qui ressortit du genre du répertoire d'exempla pour répondre à l'injonction d'efficacité (statim invenire) des instruments de travail de prédicateurs. Jean Gobi montre un fort goût pour l'abrègement des récits, ce qui n'empêche pas d'y insérer parfois une moralisation, notamment pour les fables animalières et quelques longs récits.

8. Filippe de Ferrare ( $†$ milieu du XIV ${ }^{\mathrm{e}}$ siècle), dominicain, composa son Manuel de conversation ( Liber de introductione loquendi) dans les années 1321-1347. Il était destiné à ses confrères dominicains pour leur apprendre comment se comporter et parler dans les différentes circonstances de la vie : à table, au coin du feu, en voyage, avec les malades, pour consoler une personne affligée. La diffusion de l'ouvrage a été très modeste aussi bien chez les dominicains que dans la littérature didactique postérieure.

9. La Table des exemples (Tabula exemplorum) est un recueil attribué à un franciscain français anonyme qui l'aurait composé dans les années 1270-1285. C'est un des premiers recueils à adopter l'ordre alphabétique : ses quelque 300 exempla sont répartis dans 151 rubriques (ou chapitres). Ce recueil a connu un succès certain, comme en témoignent les 22 manuscrits complets recensés par Welter dispersés dans toute l'Europe et les nombreux emprunts faits par les recueils postérieurs.

10. Bernardin de Sienne, franciscain de l'Observance (1380-1444), entré dans l'ordre à Sienne en 1402, fut envoyé prêcher dans l'Italie du Centre et du Nord de 1408 à 1427. Sa prédication (Cannarozzi 1934-1958, Delcorno 1989) rencontra un succès immense et eut une grande influence politique et sociale. Il devint vicaire général de l'Ordre des Frères Mineurs. Dès sa mort, il était considéré comme l'un des plus grands saints d'Italie, il fut canonisé dès 1450 . Ses sermons abordent tous les sujets de la vie quotidienne et, à ce titre, font une place aux animaux.

11. Le Ci nous dit (Blangez 1979-1986) a été composé dans les années 1313-1330, en ancien français (picard) par un auteur inconnu. Les 780 chapitres du Ci nous dit ont été enluminés dans le manuscrit de Chantilly (Heck 2012) et, dans une moindre mesure, dans d'autres manuscrits, mais il existe également des manuscrits dénués de toute enluminure et destinés non plus à la lecture aristocratique, mais à la prédication. Ce recueil composite inclut un bon nombre d'exempla dont certains mettent en scène des animaux.

12. Un récit exemplaire de Filippe de Ferrare fait allusion à la confection d'un médicament contre les champignons vénéneux à base de thériaque, d'excrément de coq et d'eau chaude. N. 92. 


\section{RÉSUMÉS}

À partir d'un corpus choisi de sermons et d'exempla du XIII ${ }^{\mathrm{e}}$ siècle jusqu'au début du Xve siècle, nous serons à même de saisir l'imaginaire attaché à la poule, de la comparer aux autres oiseaux et d'observer sa mise en scène dans un contexte social proche des realia. Ces textes nous éclairent également sur la place de ce volatile dans le savoir naturaliste moralisé inspiré des bestiaires et enfin sur l'allégorisation extrême de la poule et du poulet qui peuvent représenter la Vierge, le Christ et même Dieu dans quelques sermons et exempla.

Thanks to a chosen corpus of sermons and exempla from the 13th century to the 15th century, we will be able to catch the imaginary linked to the hen, to compare it with other birds, to observe its setting in a social context near to realia. These texts also shed a light on its place in the moralized naturalist knowledge, inspired by Bestiaries and finally the extreme allegorization of the hen and the kitchen used to represent Virgin Mary, the Christ or even God, in some sermons and exempla.

INDEX

Keywords : animal, hen, preaching, Middle Ages, history, rhetoric, metaphor, allegory

Mots-clés : animal, poule, prédication, Moyen Age, histoire, rhétorique, métaphore, allégorie

\section{AUTEUR}

\section{MARIE ANNE POLO DE BEAULIEU}

Directrice de recherche au CNRS, Chercheuse rattachée au Centre de recherches historiques INHA/ALHOMA, 2, rue Vivienne, 75002 Paris

polo@ehess.fr 